\title{
IMPLEMENTATION OF INTERACTIVE AUGMENTED ReAlity in 3D Assembly Design PrEsentation
}

\author{
Andrijana Bocevska and Zoran Kotevski \\ Faculty of Information and Communication Technologies, “St. Kliment Ohridski” \\ University - Bitola, Bitola, Republic of Macedonia
}

\begin{abstract}
The visual representation of a product and the role of visualization have recently become a central issue in design research. By enriching a real scene with computer generated objects, Augmented Reality, has proven itself as a valuable Human-Computer Interface in numerous application areas, such as medicine, military, entertainment and manufacturing. In this paper we propose an approach by which a user can create own $3 D$ augmented reality scenes that enable interaction between the real world and virtual assembly's components, while including an animation at the same time. For this purpose, BuildAR Pro software is employed using marker-based camera tracking, while assembly design is obtained with standard CAD system SolidWorks. The animations are developed in 3ds max software package in order to save the assembly as ive file format, which is helpful to increase the performance of scene rendering and/or viewing.
\end{abstract}

\section{KEYWORDS}

Augmented reality, Animated assembly design, Marker-based camera tracking

\section{INTRODUCTION}

Design visualization plays a critical role in achieving an accurate design. Current Computer Aided Design (CAD) systems, intended for designers and engineers, offer extremely rich modeling features and functions which increase the productivity of new assembly designs. But, they still have limitations in understanding of complex structures, since their models are presented with certain constraints on users' interactions with the design model. Although the geometric databases has been 3D for a long time, the user interaction made through keyboard, mouse and CRT/LCD display, which are only 2D devices, with CAD systems has not been significantly changed.

Augmented reality (AR) is a research field of computer science that deals with the combination of real world objects and computer generated data. Unlike Virtual Reality (VR) systems, in which users are completely immersed in the virtual environment, AR users see the virtual components and the real world co-existing in the same space. It is the goal of AR to supplement the reality, rather than completely replace it as in conventional VR applications. The AR technology provides useful information about the environment enhancing perception and interaction with the real world. The user is able to manipulate both, real objects and the virtual ones. AR provides new perspectives for user interaction with CAD tools. AR enhances the perception of assembly's components by co-location, leading to information with less perceptive ambiguities. The users have the possibility to translate, rotate and scale the overlaid assembly model by using a tangible user interface composed from indicative markers. AR helps users to comprehend space more effectively by letting the users to visualize and interact with designs in a more intuitive way. AR can be used in almost every phase of the design and manufacturing, i.e. from the initial concept and design phase, during the reviewing and evaluating of concepts and alternatives, all the way to 
the manufacturing phase. AR can also provide detailed information about maintenance procedures, in the marketing of a certain product presenting different configurations.

According to Milgram et al. [1], AR is also known as Mixed Reality (MR), as presented in Figure 1. MR aims to combine the best aspects of both, VR and AR. It refers to the entire spectrum of situations that span the continuum between VR and actual reality. According to this, MR can include AR, augmented virtuality and other mixed configurations. In MR environments, users can navigate through both, the real and virtual environments at the same time. Virtual objects are anchored into the user's real world space and augment their real world environment, making virtual interactions to appear as "real".

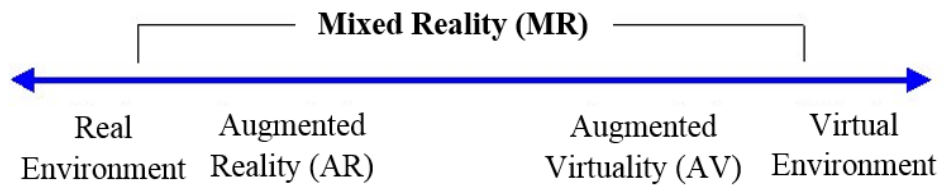

Figure 1. Simplified representation of a reality-virtuality (RV) continuum

Having characteristics of integration of virtual and real world scenes, many applications of AR are emerging in the fields of education, medical treatment, entertainment and design, to name a few. The characteristics of AR systems can be further understood from three classical and widely used criteria defined by Azuma [2], listed in the following lines:

- Combines virtual and real - AR requires display technology that allows the user to simultaneously observe virtual and real world information in a combined view. Traditional displays can show only computer-generated images, thus are insufficient for AR.

- Registered in 3D - AR relies on an intimate coupling between the virtual and the real world that is based on their geometrical relationship. This makes it possible to render the virtual content with the right placement and 3D perspective with respect to the real world scene.

- Interactive in real time - the AR system must run at interactive frame rates, such that it can superimpose information in real-time and allow user interactions.

The basic components in AR applications, as proposed by Azuma et al. [3] and Siltanen [4] are: a display, a camera and a computer with application software. Different kinds of hardware can be used to implement AR, e.g. camera phones, handheld computers, laptops, head-mounted displays (HMD) etc. Typically, a camera is attached to the display device which shows the real time camera view "through" its screen. To determine the relation between real and virtual worlds, computer vision techniques are used to find a marker in the camera image and determine the position and the pose of the camera relative to it. Once the position and the pose of the real camera are known, a digital 3D model can be overlain on the marker in the camera image. Thus, the user experiences video see-through augmented reality, seeing the real world through the real time video augmented with virtual models.

In the latest years AR has been in the research focus quite extensively, especialy in the hardware technology. In the past AR systems emerged as large and robust, but the rapid development of mobile devices has lead to the requirements of enabling small units with enough processing capacity and long lasting batteries to enable light-weight mobile AR applications. Recently, PDAs according to Pasman and Woodward [5], camera phones according to Henrysson et al. [6] and Rohs [7] and miniature PCs according to Honkamaa et al. [8] have been successfully used for AR applications. 
Today AR is divided into two parts: Location-Based AR and Vision-/Recognition-Based AR, as in [9]. Location Based AR is the concept of using GPS or other instruments, external and internal such as gyros, to determine the user's location and orientation, in order to determine what the user might be looking at, so that a relevant information could be displayed. One of the most common uses of this type of AR is to show the user a certain Point Of Interest (POI:s), for instance if the user is on a trip and wants to know about the landmarks or buildings around him, or maybe the nearest restaurant. Vision Based AR is about tracking objects or markers and provides additional information. Common use of this could be to track a marker or a poster and then display some graphical object on top of this marker or poster. In Vision Based AR there are three types of trackings that are mostly used today: 1) Marker tracking, 2) Markerless tracking / Natural Feature Tracking, 3) Markerless 3D tracking, as in [9].

Marker tracking uses markers as its tracking focus. These markers are commonly known as ARtags and have a black frame of various thickness. Inside this frame can reside various types of information, where some have pictures while others have small black squares, as commonly seen in QR-codes. The most common type is a marker that is only black and white, where the content/picture inside the black frame is composed of small black squares. The black frame is used for camera calibration to determine at what angle the marker is viewed from, and accurately calculate the correct perspective in which a graphical content should be drawn, as in [9]. The marker can be used as the exact location and graphical content will be drawn on top of it, but it can also be used for camera calibration, i.e. perspective calculation, allowing the drawing of one or multiple movable objects around the marker in the correct perspective.

Markerless tracking, also known as Natural Feature Tracking (NFT), is about tracking real world objects, most commonly pictures, posters and other printed images. These markers are different from the previous mentioned markers in that that they do not need any black frame surrounding the content and the content can be anything. However, in today's tracking technologies there are restrictions to what the content may be in order to obtain substantial tracking results. The content cannot be too simple, otherwise other content can mistakenly be tracked instead, and therefore the content should be more complex and contain many contrast points. The contrast points inside the content work as a map for the content, in such a way that the more contrast points present the more accurate is the calculated viewing angle and the distinguishing of the content uniqueness, which provides better overall tracking.

Markerless 3D tracking makes it possible to track real world objects in 3D. The technology allows for graphical content to disappear behind real objects and further create the illusion of that the virtual is coexisting in the real. This kind of tracking uses the same technology as in NFT tracking, with the addition of calculations that determine the 3D shape of the tracked object.

In addition to these three tracking types there is one more concept that can be added to all the tracking systems, and that is Virtual buttons, as in $[10,11]$. Virtual buttons allow the user to interact with the information given without touching the screen. The common way of making Virtual buttons is to mark a special area on a marker, when this special area is covered by the user, such that it cannot be observed by the camera, an option is triggered.

The aforementioned technologies may be used in various domains that require integration of some graphical representations with real world scenes. To contribute to the quite large field of such implementations, in this paper we propose an approach by which a user can create own 3D augmented reality scenes that enable interaction between the real world and the components of a virtual assembly, while including an animation at the same time. For this purpose, we employed the BuildAR Pro software and marker-based camera tracking. The assembly design is obtained 
using standard CAD system SolidWorks. The animations are developed in 3ds max software package in order to save the assembly as .ive file format, which is helpful to increase the performance of scene rendering and viewing.

The rest of the paper is organized as follows. Section 2 presents the related work and the main motivation for this research. Section 3 gives an overview for integration of CAD designs and AR applications and explains the process of real world augmentation with virtual CAD assemblies. Detailed presentation of the augmentation process is given as well. Section 4 concludes the paper and outlines directions for future work.

\section{MOTIVATION AND RELATED WORK}

In the past decade numerous research efforts implement AR paradigm as an addition to certain areas using CAD designs, but they are mainly concentrated on training applications. In this manner, Chimienti et al. [12] use AR in training operations for assistance to unskilled operators during an assembly tasks, such as of a planetary gearbox, with a help of a hand-held device. The authors also declare that these proposed guidelines of AR implementation offer an effective point for a training systems, and their general nature gives provides the flexibility to make it applicable to other fields. Compared to the training techniques used previously, the application of AR concept seems to offer a set of advantages, such as a significant reduction of time and a lower investments in human resources. The implementation of AR in training has also been investigated by Webel et al. [13] in the field of maintenance and assembly where instructions, or location dependent information, is directly linked and/or attached to physical objects. The main conclusions are that AR offers great potential for industrial training, because it can easily be integrated in teleconsultation technologies and it can be realized using standard hand-held devices. Sääski et al. [14] take a similar approach, but instead of training, they concentrate on the implementation of AR to assisting assembly work in real life production environments. The work is supported by a description of a real case experience in a tractor company in Finland. On the other hand, Gavish et al. [15] and Peniche et al. [16] combine AR with virtual reality (VR) to improve training and assembly processes in manufacturing mechanical parts. Both researches present similar outcomes and conclusions, such as increased training efficiency, enhanced acquisition of skills, help for trainees to develop a better mental model of the required task. This survey revealed that the current implementations of AR use static part or assembly designs and that animations are not considered. Thus, in this research, we concentrate on the implementation of animated assembly designs, but not only for training or production purposes, but in marketing and presentational purposes as well.

To the best of our knowledge this is the first effort to employ animated assemblies in AR applications, which further opens more application areas of CAD design in the technologies incorporating AR. 3D assembly animation has emerged as a benchmark for the mechanical engineering worldwide. It is increasingly being used to test products and implement real-time changes for maximum performance and efficiency.

In this paper, we employ a Marker-based AR technique to enrich a specific real world scene with an assembly. Besides the fact that the assembly can be observed from different angles and views, it integrates an animation as well, that provides detailed presentation of assembly's components in an "explode" animated views.

For this purpose, first we used BuildAR Pro application because it is designed to allow users to easily add virtual objects into a live video of the real world, where AR scene can be created without any programming at all. Normally, the creation of AR applications requires significant programming experience. In order to use this application it is necessary to choose tracker type, 
select video source, add a visual tracking marker and finally add virtual content to the real printed markers. The application interface allows users to train their own markers, change camera threshold values, and add video and audio content and other features if required. The software also supports a variety of other content types, such as 3D Models, 2D Images, Quick Time Movies, Text, Primitive Shapes and Sounds.

\section{AN APPROACH FOR INTEGRATION OF AN ANIMATED CAD ASSEMBLY IN AN AR APPLICATION}

As mentioned earlier, we implemented marker-based technology to integrate animated CAD assembly designs in AR scenes. A marker-based paradigm uses square patterns (markers) that are simply printed on a piece of paper and displayed in the camera viewing field. The AR software uses computer vision technology algorithms to recognize the marker patterns and calculate the position of the real camera relative to the printed pattern. Once the position of the real camera is known, then an assembly's components can be drawn relative to the real marker. Assembly's components are virtual objects that will appear to be attached to the markers, creating augmented reality experience.

Printed markers usually are black and white squares with thick borders, and a unique pattern in the center. Different markers are recognized by their different patterns inside the borders. Hence, quite important moment here is that there should be enough white space around the outside of the black square so that the marker can be easily found by the computer vision software. The pattern in the middle of the square should be twice the width of the border of the marker. The BuildAR Pro software is able to use two different marker types (hiro and kanji), as presented in Figure 2, but anyone is allowed to create own marker, if these two do not fulfill the application requirements.
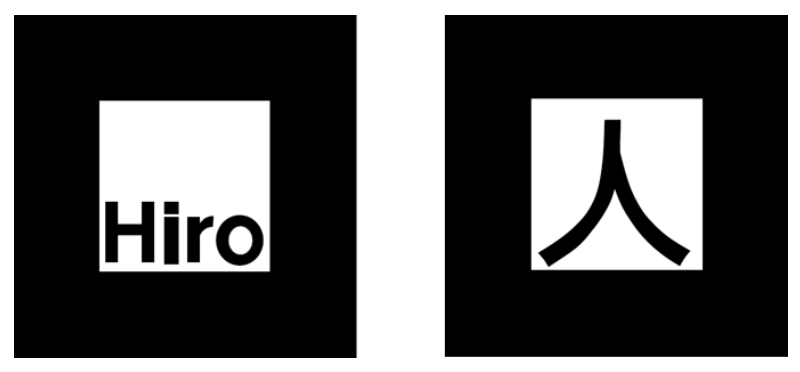

Figure 2. Examples of the two types of AR markers (hiro and kanji)

For the integration of a CAD system and augmented reality we decided to engage SolidWorks as a powerful 3D design solution for rapid creation of parts, assemblies, and 2D drawings, and BuildAR Pro as a tool for fast building of AR scenes, while providing a live video viewing with 3D virtual models on images and markers. BuildAR Pro uses marker-based camera tracking, which means that the assembly's components appear attached to physical printed marker. Augmented reality scene can be built by creating a set of markers and a set of 3D models. Assembly's components can be created in almost any modelling program or downloaded from the Internet. BuildAR Pro also provides an easy to use graphical user interface that simplifies the process of creating AR scenes, meaning that there is no need for programming skills or expertizes in computer science to create the real/virtual scenes. BuildAR Pro is a product of the Human Interface Technology Lab NZ, University of Canterbury, located in Christchurch, New Zealand. The approach for integration of a CAD assembly in AR application involves several activities that we further elaborate in more details. 
Activity 1. Generation of patterns in BuildAR Pro. Before tracking patterns can be produced, the images have to be previously created in an image editing software tool and saved as a bitmap file with 16 bit color depth. The created markers for this approach use text as an image.

Activity 2. Loading the $3 D$ model over the predefined marker. In this step we assume that the assembly design has already been developed with a CAD system, for which in this case we use SolidWorks. In order to use the created 3D CAD models in BuildAR Pro they must be converted in one of the supported file formats, which include 3DS, LWO, OBJ, STL, FLT. The recommended file format is the OpenSceneGraph .ive file format. Some other file formats can be used as well, but the quality of the imports for these other formats varies considerably, and sometimes they can produce strange normals, or missing textures and/or animations. Because our main intention concerning BuildAR Pro is to display an animation by identifying a marker, the animation needs to be prepeared in $3 \mathrm{ds}$ max. For that purpose we imported the assembly from SolidWorks in 3ds max with .sldasm file format. After the creation of the animation using the OpenSceneGraph plugin we export the animation in ive file format, given that this format is better supported by BuildAR Pro, although we can use the .fbx file format as well. Nevertheless, we can also create the animation directly in SolidWorks, but certain limitations are imposed, since in this manner the model can only be exported as a video file in the .avi format, which destroys the possibility to represent a native $3 \mathrm{D}$ animation.

Activity 3. Moving, rotating and scaling the model. To adjust the way the 3D model appears on the marker there is a built in manipulation control panel, where the new positions, rotations and scales can be typed manually, or simply by clicking on the 3D object in the live view while using the 3D widget buttons on the displayed toolbar. After the import of the 3D model, there is a Normalize option at a user's disposal, by means of which the program automatically displays the 3D model to best fit the preview window. At the same time the scene tree shows which markers and $3 \mathrm{D}$ objects currently make up the scene. The tree is a list of markers, each with an object underneath. Markers and object can be added or deleted from the scene tree. If adding more markers and $3 \mathrm{D}$ objects is required to additionally augment the real scene these steps must be repeated.

Activity 4. Saving and loading the augmented reality scene. After editing, the scene can be saved to an XML file.

\subsection{The augmentation process steps}

The whole augmentation process consists of multiple consecutive steps to realize the task of CAD assembly placement over the predefined marker. These steps occur as follows:

1. The video camera captures video of the real world and sends it to the computer.

2. The live video image is turned into a binary (black and white) image based on a lighting threshold value.

3. This image is then searched for square regions. BuildAR Pro software finds all the squares in the binary image.

4. For each square, the pattern inside the square is captured and matched against some preentered pattern templates.

5. The BuildAR Pro software then uses the known square size and pattern orientation to calculate the position of the real video camera relative to the physical marker. BuildAR Pro software computes the transformation matrix that describes the position, rotation, and size of an object. 
6. The virtual object is thereafter located in relation to that marker. Thus, the user experiences augmented reality, seeing the real world through the real time video with virtual models.

The diagram in Figure 3 presents the image processing that is conveyed in the background in order to produce the AR experience, while Figure 4 reveals an example of a sample marker in a video frame with and without the animated virtual object.

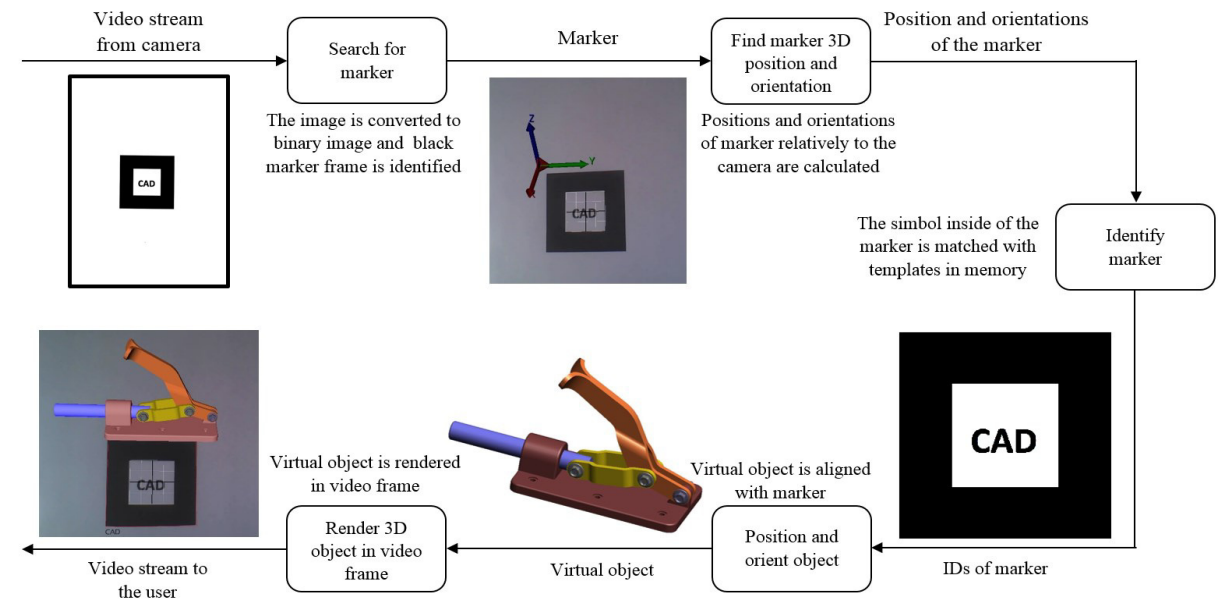

Figure 3. The process of video-based marker detection and overlay of virtual object

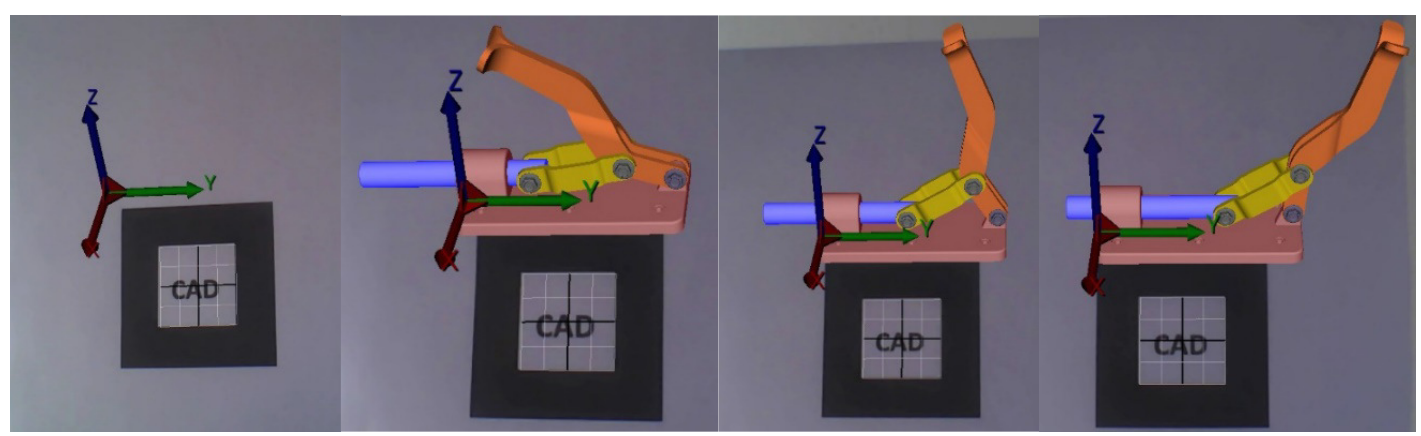

Figure 4. Sample marker in frame with and without a virtual animated 3D assembly

\section{CONCLUSION AND FUTURE WORK}

Marker based augmented reality technologies represent quite useful tools that can be used for creation of interactive visualizations of 3D CAD models. The advantages of this type of application are the opportunities to display a 3D CAD model in a real world scene, while enabling the user to interact with it in the manner of translation, rotation and scaling of the overlaid model by the simple usage of a printed marker placed in the camera viewing field. Presented visual graphics enable viewers to comprehend the core concept and features behind the design of the assembly, even if they are not from a technical background. Besides, this paper presents the possibilities to use augmented reality technologies in CAD assembly design supplemented with animations i.e. the possibilities to view the virtual object and its constituent parts in the real world using a marker based AR system. Such product animations also provide better overview of the final product, its performance and overall efficiency. The representation provides an opportunity to demonstrate a virtual prototype to potential clients and help them to understand the product and 
its features. It gives presentations an added flavor of reality which is otherwise downrightly impossible by the conventional methods. To realize this task, for the translation of the CAD model and its import to the AR software, the 3ds max software was used as a middleware for the purpose to convert the SolidWorks's .sldasm file format to an animated ive file. The animation in $3 \mathrm{ds}$ max was exported to the .ive file format by the utilization of the OpenSceneGraph plugin, given that this format is better supported by AR software. Our opinion is that this approach plays an important role in design visualization and can be used in achieving proper understanding of the design. As future research directions we will concentrate our efforts in developing an implementation of this idea in the area of cataloging machine parts and/or assemblies. Similarly to the work of Patkar et al. [17] and Pasman and Woodward [5], the idea is to store markers, as well as their linked 3D models, in a database that is accessed via Internet using a software similar to BuidAR (desktop or mobile), while the markers are printed in a single or multiple page catalog or presented in a web interface. In this manner, the complex 3D parts and assemblies would be easily disseminated among interested parties, while at the same time reducing the cost of marketing actions. Another implementation that we intend for our future work is to print the corresponding marker on the assembly package, so that a customer can be informed of the product details without opening the package. To conclude our future intentions we will explore the possibilities to augment the whole AR animated assembly presentation with audio explanations of the specific functions and the advantages of the referred product.

\section{REFERENCES}

[1] Milgram, P., Takemura, H, Utsumi, A. \& Kishino, F. (1995). Augmented reality: A class of displays on the reality-virtuality continuum. Photonics for industrial applications, pp. 282-292. International Society for Optics and Photonics.

[2] Azuma, R.T. (1997). A survey of augmented reality. Presence: Teleoperators and Virtual Environments, 6(4):355-385.

[3] Azuma, R., Baillot, Y., Behringer, R., Feiner, S., Julier, S. \& MacIntyre, B. (2001). Recent advances in augmented reality. IEEE Computer Graphics and Applications, 21(6), 34- 47.

[4] Siltanen, S. (2012). Theory and applications of marker-based augmented reality. Julkaisija Utgivare Publisher, Copyright @ VTT 2012 ISBN: 978-951- 38-7450.

[5] Pasman, W., Woodward, C. (2003). Implementation of an augmented reality system on a PDA. Proceeding of the Second IEEE and ACM International Symposium on Mixed and Augmented Reality (ISMAR), pp. 276-277, Tokyo, Japan.

[6] Henrysson A, Billinghurst M, Ollila M, (2005). Virtual object manipulation using a mobile phone”, Proc. 15th International Conference on Artificial Reality and Telexistence (ICAT 2005), Dec 5th 8th, 2005, Christchurch, New Zealand, pp. 164-171.

[7] Rohs M, (2006). Marker-Based Embodied Interaction for Handheld Augmented Reality Games, Proceedings of the 3rd International Workshop on Pervasive Gaming Applications (Per-Games) at PERVASIVE 2006, Dublin, Ireland.

[8] Honkamaa, P., Siltanen, S., Jäppinen, J., Woodward, C., \& Korkalo, O. (2007). Interactive outdoor mobile augmentation using markerless tracking and GPS. In Proc. Virtual Reality International Conference (VRIC), Laval, France (pp. 285-288).

[9] Geroimenko, V., (2012) Augmented Reality Technology and Art: The Analysis and Visualization of Evolving Conceptual Models. 16th International Conference on Information Visualization, pp. 445 453.

[10] Newman, J., Ingram, D., \& Hopper, A. (2001). Augmented reality in a wide area sentient environment. In Augmented Reality, 2001. Proceedings. IEEE and ACM International Symposium on (pp. 77-86). IEEE..

[11] Yuan, M. L., Ong, S. K., \& Nee, A. Y. C. (2008). Augmented reality for assembly guidance using a virtual interactive tool. International Journal of Production Research, 46(7), 1745-1767.

[12] Chimienti, V., Iliano, S., Dassisti, M., Dini, G. \& Failli, F. (2010). Guidelines for implementing augmented reality procedures in assisting assembly operations. International Precision Assembly Seminar, pp. 174-179. Springer Berlin Heidelberg. 
[13] Webel, S., Bockholt, U., Engelke, T., Peveri, M., Olbrich, M. \& Preusche, C. (2011). Augmented Reality training for assembly and maintenance skills. BIO Web of Conferences, Volume 1, paper 97. EDP Sciences.

[14] Sääski, J., Salonen, T., Hakkarainen, M., Siltanen, S., Woodward, C. \& Lempiäinen, J. (2008). Integration of design and assembly using augmented reality. International Precision Assembly Seminar, pp. 395-404. Springer US.

[15] Gavish, N., Gutierrez, T., Webel, S., Rodriguez, J. \& Tecchia, F. (2011). Design guidelines for the development of virtual reality and augmented reality training systems for maintenance and assembly tasks. BIO web of conferences, Volume 1, paper 29. EDP Sciences.

[16] Peniche, A., Diaz, C., Trefftz, H. \& Paramo, G. (2012). Combining virtual and augmented reality to improve the mechanical assembly training process in manufacturing. Proceedings of the 6th WSEAS international conference on Computer Engineering and Applications, and Proceedings of the 2012 American conference on Applied Mathematics, pp. 292-297. World Scientific and Engineering Academy and Society (WSEAS).

[17] Patkar, R.S., Singh, S.P., \& Birje, S.V. (2013). Marker based augmented reality using Android OS. International Journal of Advanced Research in Computer Science and Software Engineering (IJARCSSE), 3(5), 64-69. 